\title{
EFFICACY OF FLAXSEED CRACKERS IN AMELIORATING CLINICAL AND BIOCHEMICAL HYPERANDROGENISM IN YOUNG ADULT SOUTH INDIAN WOMEN DIAGNOSED WITH PCOS
}

\author{
Jeyamani Divya Christodoss ${ }^{1 *}$, Priya D John $\mathrm{MD}^{2}$, Vasantha Esther Rani ${ }^{3}$
}

\author{
${ }^{1}$ Assistant Professor, Loyola College, Chennai \\ ${ }^{2}$ Assistant Professor and Senior Clinician, Emory University, GA, USA \\ ${ }^{3}$ Head and Professor, Fatima College, Madurai \\ Received - July 18, 2020; Revision - September 17, 2020; Accepted - December 08, 2020 \\ Available Online - March 25, 2021
}

DOI: http://dx.doi.org/10.18006/2021.9(Spl-1-GCSGD_2020).S116.S124

\section{KEYWORDS \\ Young adult women \\ Reproductive health \\ PCOS}

Flaxseeds

SHBG

Testosterone

Insulin

FG score

Menstrual cycle

\begin{abstract}
Young women of reproductive age are more prone to hormonal/endocrine disorders, the most common being Polycystic Ovarian Syndrome (PCOS). Research in different cultural contexts to enhance the physical, emotional, social, and reproductive health of women has been the focus of several scientific studies. High lignan foods reduce the bioavailability of free testosterone through increasing Sex hormone Binding globulin (SHBG) levels. A randomized single-blind placebo-controlled trial was carried out among women of 20 - 25 years from a single on-campus hostel facility in Chennai, India based on the Rotterdam criteria of PCOS diagnosis. The results showed a statistically significant lower serum free testosterone concentrations $(1.02 \pm 0.428 \mathrm{ng} / \mathrm{dl})$ in the flax group compared to the placebo $(1.64 \pm 0.504 \mathrm{ng} / \mathrm{dl})$, after the study period. Similarly, it was noted that the lowered levels of Sex hormone Binding Globulin (SHBG) were elevated to $15.22 \pm 14.593 \mathrm{nmol} / \mathrm{L}$ as compared to only $9.64 \pm$ $5.124 \mathrm{nmol} / \mathrm{L}$ in the placebo though not statistically significant, thus creating a promising platform for improving the imbalances in the hormone levels. Also, elevated serum insulin concentration was lowered in the flax group $(15.67 \pm 6.928 \mu \mathrm{U} / \mathrm{mL})$ than the control group $(19.09 \pm 8.526 \mu \mathrm{U} / \mathrm{mL})$. The FG score significantly reduced in the flax group thus alleviating the clinical manifestation of hirsutism. Lowering of free testosterone, serum insulin, and elevation of serum SHBG levels is attributed to the phytoestrogen (SDG) present in flaxseeds and can be an ideal alternate replacing the conventional drugs for PCOS.
\end{abstract}

* Corresponding author

E-mail: divyajc@yahoo.com (Jeyamani Divya Christodoss)

Peer review under responsibility of Journal of Experimental Biology and Agricultural Sciences.

Production and Hosting by Horizon Publisher India [HPI] (http://www.horizonpublisherindia.in/).

All rights reserved.
All the articles published by Journal of Experimental Biology and Agricultural Sciences are licensed under a Creative Commons Attribution-NonCommercial 4.0 International License Based on a work at www.jebas.org.

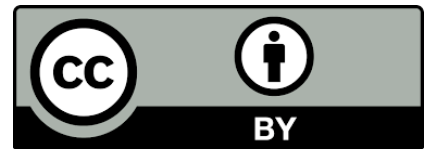




\section{Introduction}

Polycystic Ovarian Syndrome (PCOS) was first characterised with significant clinical implications like menstrual irregularities, hirsutism, infertility with the suboptimal obstetrical outcome along with metabolic imbalances such as impaired glucose tolerance, type 2 diabetes mellitus, dyslipidemia, coronary artery disease, and endometrial hyperplasia on a long term basis (Stein \& Leventhal, 1935).

PCOS is a commonly prevalent endocrine disorder diagnosed in women with features related to ovulatory and menstrual irregularities, hyperandrogenaemia with manifested clinical features such as hirsutism, acne, and androgenic alopecia, along with polycystic ovarian morphology (Azziz et al., 2006). It is often associated with metabolic aberrations leading to insulin resistance and compensatory hyperinsulinemia, which is the main factor responsible for altered androgen production and metabolic dysregulation (Escobar et al., 2005).

PCOS affected 116 million women (3.4\%) all over the world as estimated by World Health Organization (WHO) in 2012 (Vos et al., 2012) with a high global variability ranging from $2.2 \%$ to $26 \%$ (Vidya Bharathia et al., 2017). This wide range of prevalence rates across the globe is a clear indication of the fact incidence of PCOS may vary between different races and ethnicities (Li et al., 2013, Chang et al., 2016, Huang \& Yong, 2016). Based on the ethnicity, it is reported that among the Asian population, there is an increased manifestation of hirsutism with early onset of symptoms, and increased risk of insulin resistance and metabolic risk factors associated with Caucasians (Kim \& Choi, 2019). In India, the prevalence of PCOS ranges from 3.7 to 22.5 percent based on the population studied and the criteria adopted for diagnosis (Ganie et al., 2019) and middle and high-income groups of the urban sector show higher prevalence than the rural population (Balaji et al., 2015; Vidya Bharathia et al., 2017). Also, it was observed that among women from the South Indian union territory of Pondicherry, about $26 \%$ of them had hirsutism, 94\% had oligomenorrhea, $80 \%$ had ovarian morphological changes, and $6 \%$ had amenorrhea, which demonstrates a high correlation between menstrual irregularity, PCOM and clinical features of PCOS (Sheelaa \& Radha, 2014).

PCOS is characterized by metabolic and ovarian dysfunction. Metabolic derangements are known to respond to lifestyle modifications, which are advocated as the first line of treatment for PCOS, which includes changes in dietary pattern, exercise level, and behavioral therapies (Ebrahimi et al., 2017). Obesity is common among women with PCOS. Predominantly 50\% of women with PCOS are obese, and lowering body weight is proven to be supportive in normalizing hormonal levels and clinical

Journal of Experimental Biology and Agricultural Sciences http://www.jebas.org symptoms (Gambineri et al., 2002). High fiber foods have been known to reduce weight by several mechanisms, including promoting satiety, decreasing absorption of macronutrients, and alteration of gut hormones (Slavin, 2005).

While lifestyle modification is a pertinent recommendation for PCOS, decreasing insulin resistance by weight loss alone is difficult. Metformin, commonly used as an antidiabetic drug, works on insulin resistance and has been used in PCOS extensively. While metformin was shown to reduce serum insulin levels, it has not been shown to reliably restore hirsutism, androgen levels, or menstrual cycles, which are common clinical symptoms in PCOS (Johnson, 2014). Metformin has also been proven to show a higher incidence of gastrointestinal side effects, thus leading to drug discontinuation (Costello et al., 2007).

Interestingly extensive medicinal herbs have gained popularity as an ideal alternative remedy for disease prevention in the past few decades (Legro et al., 2013). Earlier scientific studies have indicated that high fiber foods, specially lignan rich, have decreased serum testosterone concentration by increasing their exertion in the enterohepatic circulation (Adlercreutz et al.,1987) and they also lower the circulation of bioavailable free testosterone by enhancing the levels of SHBG (Low et al., 2005).

Functional foods or Nutraceuticals are classified as foods that have been proven scientifically to have health benefits or diseasepreventing properties along with the fundamental nutritional properties present in them. Whole flaxseed, flax meal, and milled flax have been found to have many health-promoting and diseasepreventing properties (Bloedon \& Szapary, 2004). The flaxseed (Linum usitatissiumum) is proven to be a rich source of the biologically active constituents made up of omega 3 fatty acid namely Alpha Linolenic Acid (ALA), phytoesterogenic lignans, namely Secoisolariciresinol Diglycosidic (SDG), and dietary fibers (Yari et al., 2016). It has been proven that flaxseed is also a functional ingredient used in baking applications contributing to improved tenderness and crunchiness of the product. The lignan present in flaxseed is found to be not affected by the high temperatures used in baking while incorporated into baked products and has gained popularity as a bakery ingredient (Lipilina \& Ganji, 2009).Given this background of an increased clinical manifestation of PCOS in a South Indian population, this present study is focussed on assessing the efficacy of functional foods in ameliorating the clinical and biochemical manifestation of PCOS among young adult women from an urban population in South India. The study aims to assess the impact of flaxseed crackers, a baked product, on the metabolic and hormonal status among young women with $\mathrm{COS}$ and create awareness on the importance of healthy dietary and lifestyle habits in improving the prognosis of the disorder. 


\section{Materials and Methods}

\subsection{Study design}

The design of this pilot study was a single-blind with two arms, placebo controlled randomized trial consisting of an intervention arm supplemented with flaxseed cracker (30g/day) and plain wheat cracker $(30 \mathrm{~g} / \mathrm{day})$ in the control arm. The present study protocol evaluated the efficacy and the safety of milled flaxseed crackers supplementation on the clinical and biochemical key features among young adult women of South Indian origin in the age group of 20-25 years. Milled flaxseed was purchased from the local market and baked into crackers weighing $15 \mathrm{~g}$ each and was stored in an airtight, cool, and dark container before the study's onset. Similarly, wheat crackers weighing $15 \mathrm{~g}$ each were prepared and stored for the control arm. The dosage of $30 \mathrm{~g} /$ day of flaxseed was decided based on the findings of previous systematic reviews and meta-analyses (Sartang et al., 2017, Rahimlou et al., 2019). And it was taken care of to see that greater than $50 \mathrm{~g} /$ day was avoided to prevent any side effects (Carraro et al., 2012). The study design and the methods were approved by the Independent Ethics Committee (IEC), Ethica Norma for Human Clinical Trial and all participants provided signed informed consents.

\subsection{Participants}

Participants for the study were selected based on Rotterdam criteria of diagnosis for PCOS (Wang \& Mol, 2017) from a single on-campus hostel facility in the metro of Chennai, Tamil Nadu, South India. Willing participants for the study were assessed by a gynecologist for identifying clinical symptoms of PCOS ( $\mathrm{mFG}$ score) and PCOM using the ultrasound technique and evaluated if they met the Rotterdam criteria. According to the Rotterdam criteria, any two of the following symptoms were used for inclusion of the subjects, that is, irregular periods 8-9 cycles/year, signs of hyperandrogenism with a FG score greater than 8 (Aswini \& Jayapalan, 2017), serum testosterone levels > $180 \mathrm{ng} / \mathrm{dl}$ and polycystic ovaries in the ultrasound. Totally 24 participants within the age range of 20-25 were assigned into two arms of the study. The experimental arm (12 participants) received milled flaxseed in the form of crackers ( $30 \mathrm{~g})$, and the placebo arm (12 participants) received wheat crackers $(30 \mathrm{~g})$. The intervention was administered for 5 months. Recruitment of subjects was done based on a randomization schedule using an online randomization generator. Biochemical levels for serum SHBG, Total testosterone, and Insulin concentrations were analyzed in a NABL accredited laboratory before and after the supplementation period. Free testosterone and bioavailable testosterone levels are deduced from estimated total testosterone and albumin levels in the blood (Figure 1).

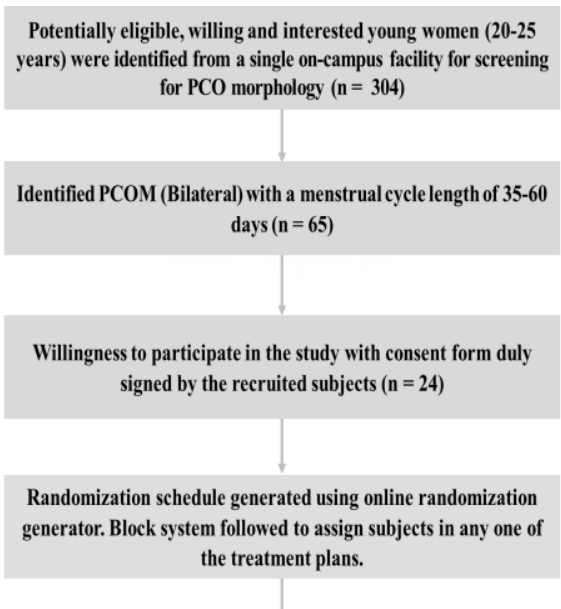

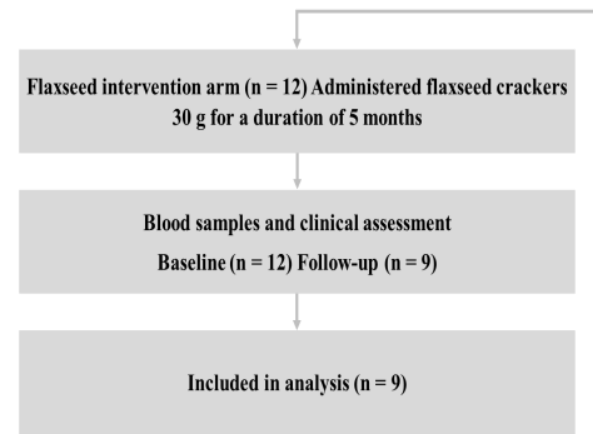

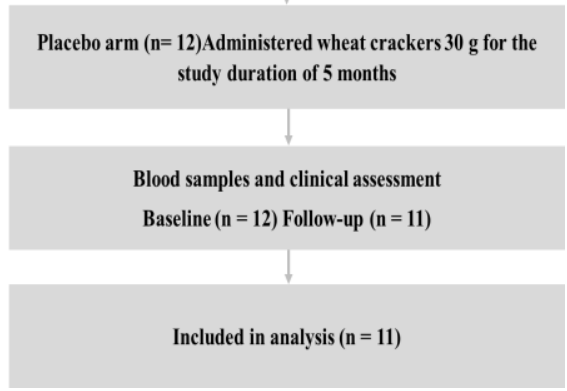

Figure 1 Systematic representation of study and participant recruitment

Journal of Experimental Biology and Agricultural Sciences

http://www.jebas.org 


\subsection{Inclusion criteria}

Female participants aged between 20 and 25 years (both inclusive) residing in an on-campus hostel facility in the metro of Chennai. BMI between 18.5 and 40 (both inclusive). Diagnosed for any two of the following symptoms as per Rotterdam criteria, that is, Oligomenorrhea/Amenorrhea (two years after menarche) i.e., irregular periods 8-9 cycles/year, signs of hyperandrogenism (Clinical or Biochemical), Hirsutism (FG score greater than 8), Testosterone levels $>6.2 \mathrm{nmol} / \mathrm{L}$, Polycystic ovaries in ultrasound, Signs of hyperinsulinemia: Obesity, Acanthosis Nigricans. Ability to understand and willingness to sign statements of informed consent.

\subsection{Exclusion criteria}

Women on other medications for PCOS such as oral contraceptives, metformin, spironolactone, cimetidine, cyproterone acetate, cyproheptadine, and glucocorticoids. Any known history of hypersensitivity or idiosyncratic reaction was observed to any part of the flaxseed and other members of the Linaceae plant family. Women are known to have past episodes of allergies with a clinical significance which includes drug allergies or bronchial asthma due to allergic reaction. Women with known occurrences of heart and pulmonary diseases, liver and renal disorders, gastrointestinal disturbances and gastric surgery, hormonal imbalances, skin allergies, neurological and psychiatric disorders will be excluded from the study. Known history of any major illness and presence of any significant organ abnormalities. Women administered with over the counter (OTC) and prescribed medications, enzyme modifying medication, and systemic medication taken within the last 30 days before the start of the study were not included.

\subsection{Outcome measures for the study}

Primary biochemical outcome measures were serum insulin level, Total testosterone, SHBG, free testosterone, and bioavailable testosterone, along with hyperandrogenic clinical variables such as hirsutism and menstrual cycle status. Clinical evaluations were determined using a modified Ferriman-Gallwey score (Aswini \& Jayapalan 2017).
Menstrual cycle regularity was evaluated using a questionnaire and personal interviews before and after the study period.

\subsection{Laboratory methods}

Blood samples from the participants were collected in the morning after an overnight fast before and end of the study period. Quantitative determination of serum total testosterone concentration and serum insulin concentration were done using Electro-Chemiluminescence immunoassay and serum SHBG concentration was analyzed using Chemiluminescence immunoassay in a NABL accredited laboratory in the metro of Chennai.

\subsection{Statistical analysis}

The data collected were expressed as Mean \pm Standard Deviation (SD). Statistical analyses were done with the software IBM SPSS version 20 with a p-value of $<0.05$ being statistically significant. The statistical tools, independent sample t-test, and t-test for paired samples compared the parameters between the two groups before and after supplementation of the flax crackers and placebo. Descriptive statistics like frequency and percentage were used to identify the sample characteristics.

\section{Results}

In the present pilot study, 24 participants were recruited, and among these, $20(83 \%)$ of them completed the study trial. Three participants from the flaxseed group and one participant from the placebo group discontinued the study citing personal reasons of shifting to their hometown, and non-compliance of the participants throughout the study protocol. More than $90 \%$ of the flax crackers were consumed by the participants in the intervention group. Compliance with the study intervention was ascertained by scheduled periodic phone calls and in-person visits to the study participants. None of the participants complained of any side effects related to flax consumption such as bloating and abdominal pain. The baseline characteristics of participants in both groups are presented in Table 1. It was noted that there was no significant difference for BMI (Table 1) in both baseline and final values for both the intervention and control groups.

Table 1 Subject Characteristics and key PCOS features

\begin{tabular}{|c|c|c|c|c|}
\hline \multirow{2}{*}{ Characteristics } & \multicolumn{2}{c|}{ Flax arm } & \multicolumn{2}{c|}{ Control arm } \\
\hline Age $($ years $)$ & $21.44 \pm 1.065$ & $21.44 \pm 1.065$ & $21.72 \pm 0.962$ & $21.72 \pm 0.962$ \\
\hline BMI $\left(\mathrm{kg} / \mathrm{m}^{2}\right)$ & $27.42 \pm 6.856$ & $27.24 \pm 7.021$ & $25.55 \pm 2.989$ & $25.73 \pm 3.19$ \\
\hline FG score & $15.22 \pm 4.871$ & $11.11 \pm 2.766$ & $11.45 \pm 2.74$ & $11.36 \pm 2.99$ \\
\hline Total Testosterone $(\mathrm{nmol} / \mathrm{L})$ & $1.26 \pm 0.333$ & $1.29 \pm 0.314$ & $1.60 \pm 0.588$ & $1.81 \pm 0.560$ \\
\hline Free Testosterone $(\mathrm{ng} / \mathrm{dl})$ & $1.02 \pm 0.017$ & $1.02 \pm 0.18$ & $1.18 \pm 0.481$ & $1.52 \pm 0.426$ \\
\hline SHBG $(\mathrm{nmol} / \mathrm{L})$ & $13.66 \pm 7.246$ & $15.27 \pm 3.620$ & $16.70 \pm 8.991$ & $9.73 \pm 4.848$ \\
\hline Insulin $(\mu \mathrm{U} / \mathrm{mL})$ & $16.16 \pm 5.406$ & $15.57 \pm 6.603$ & $15.44 \pm 4.704$ & $19.01 \pm 8.107$ \\
\hline
\end{tabular}
Values are actual representing Mean \pm SD

Journal of Experimental Biology and Agricultural Sciences http://www.jebas.org 
It was observed that participants supplemented with flax crackers for 5 months had a statistically significant lower serum free testosterone concentration $(1.02 \pm 0.428 \mathrm{ng} / \mathrm{dl})$ compared to the placebo $(1.64 \pm 0.504 \mathrm{ng} / \mathrm{dl})$, with $\mathrm{t}=-2.897, \mathrm{p}=0.010(<0.05)$ after the study period (Figure 2). Serum total testosterone concentrations in the flax group $(1.33 \pm 0.500 \mathrm{nmol} / \mathrm{L})$ was lowered as compared to the control $(1.73 \pm 0.467 \mathrm{nmol} / \mathrm{L})$ though not statistically significant, $\mathrm{t}=-1.818, \mathrm{p}=0.086(>0.05)$ (Figure $3)$.

SHBG, which was lowered at the beginning of the study, was elevated to $15.22 \pm 14.593 \mathrm{nmol} / \mathrm{l}$ as compared to the placebo being only at $9.64 \pm 5.124 \mathrm{nmol} / \mathrm{l}$ after the period of supplementation (Figure 4). Though this is not statistically significant, $\mathrm{t}=1.189, \mathrm{p}=0.250(>0.05)$, it does create a promising platform for improving the imbalances in the hormone levels by increasing the serum levels of SHBG, which in turn binds with the free circulating testosterone rendering it biologically inactive.

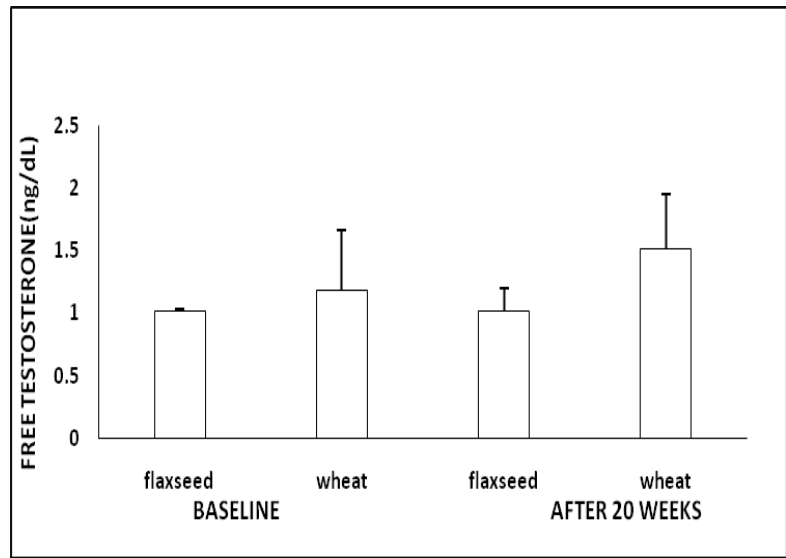

Figure 2 Free Testosterone concentrations before and after the supplementation (Data expressed as mean $\pm \mathrm{SD}$; statistically significant at 0.05 )

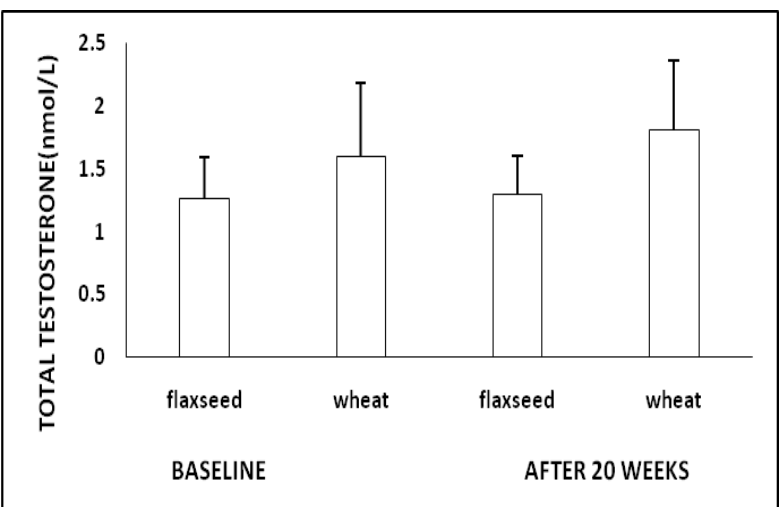

Figure 3 Serum Total Testosterone concentrations before and after the supplementation (Data expressed as mean $\pm \mathrm{SD}$; statistically significant at 0.05 )

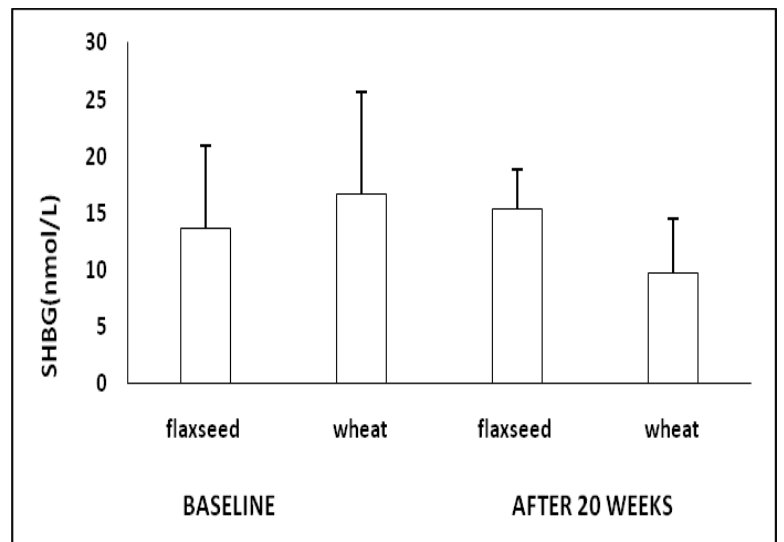

Figure 4 Serum SHBG concentration before and after the supplementation (Data expressed as mean $\pm \mathrm{SD}$; statistically significant at 0.05 )

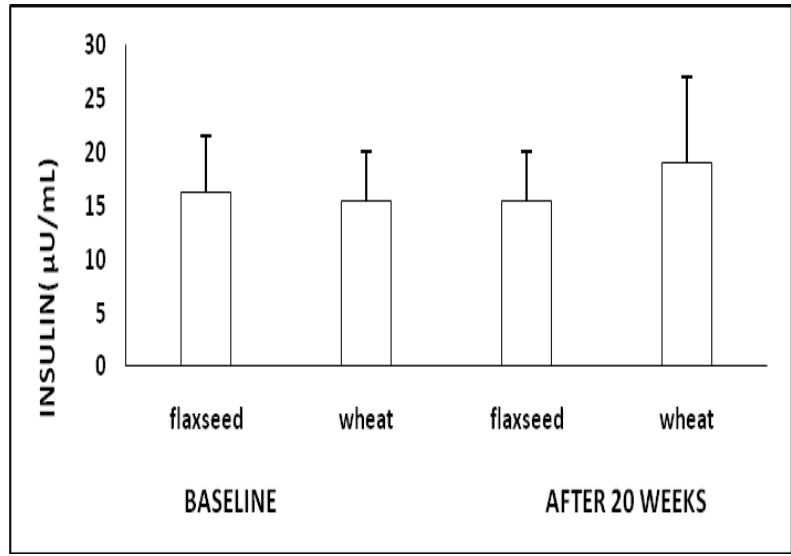

Figure 5 Serum Insulin concentrations before and after supplementation (Data are expressed as mean $\pm \mathrm{SD}$; statistically significant at 0.05 )

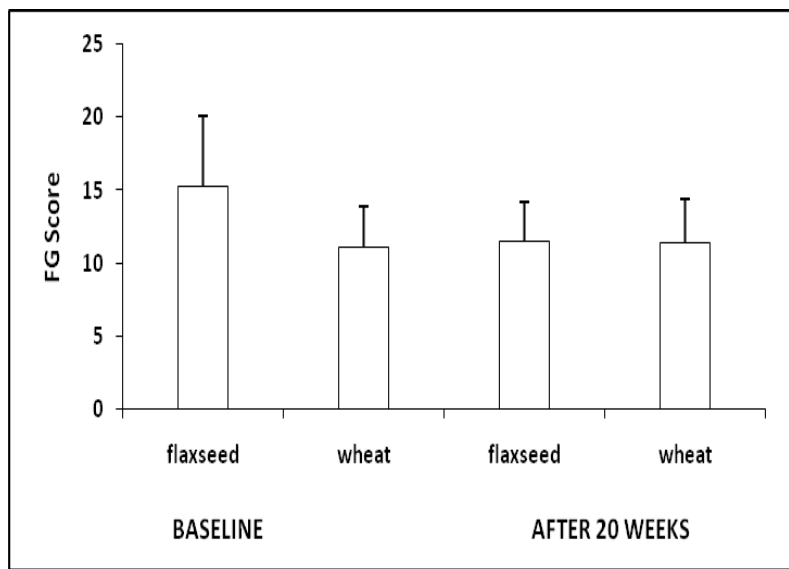

Figure 6 FG score before and after the supplementation (Data expressed as mean $\pm \mathrm{SD}$; statistically significant at 0.05 .) 
Table 2 Menstrual cycle status of participants before and after the study period

\begin{tabular}{|c|c|c|c|c|}
\hline \multirow{2}{*}{ Menstrual regularity status } & \multicolumn{2}{c}{ Flax $(\mathrm{n}=9)$} & Placebo $(\mathrm{n}=11)$ \\
& Beginning & End & $5(45.5 \%)$ & $7(77.8 \%)$ \\
\hline Regular n (\%) & $4(44.4 \%)$ & $2(22.2 \%)$ & $6(54.5 \%$ & $4(36.4 \%)$ \\
\hline Irregular n (\%) & $5(55.6 \%)$ & & \\
\hline
\end{tabular}

Various scientific studies have established the inverse relationship between SHBG and insulin concentration. It was noted that the elevated serum insulin concentration was lowered in the flax group $(15.67 \pm 6.928 \mu \mathrm{U} / \mathrm{mL})$ as against the control group $(19.09 \pm 8.526$ $\mu \mathrm{U} / \mathrm{mL}$ ) (Figure 5). At baseline, $55.6 \%$ of the participants in the flax group had menstrual irregularity. At the end of the study period, menstrual regularity increased among the participants in the flax group $(77.8 \%)$ in contrast to the control group (63\%) (Table 2).

\section{Discussion}

Young adulthood is the prime period in one's lifetime, a time of peak strength, physical performance, and in women, it is also the period of peak fertility, thus necessitating the need for healthy food habits, physical activity, and sleep pattern to augment a sound reproduction during this phase of life.

As shown in Figure 6, the FG score reduced in the flax group before $(15.22 \pm 5.166)$ and after $(11.11 \pm 2.934)$ supplementation and was found to be statistically significant in comparison to the placebo at a 5\% level of significance $(\mathrm{p}=0.043)$. Thus, the intervention with flax crackers proves to be a strong contributor in alleviating the clinical manifestation of hirsutism among young women with PCOS. Increased globalization and spearheading various workforces in different sectors of occupations have made women play a pivotal role in society. Predominantly young adult women of reproductive age are more prone to hormonal and metabolic disorders and the most common among them is Polycystic Ovarian Syndrome.

Flaxseed is a promising functional food ingredient that is used extensively in baked foods, fruit juices, dairy and dairy products, extruded pasta products, and meat products. The very fact of consuming flaxseed in the form of whole seed, milled flour, and roasted seed, provide basic nutrition coupled with various health benefits qualifying it to be considered as a functional food (Goyal et al., 2014). Several other scientific studies have proven its efficacy in decreasing androgen levels and normalizing lipid levels, though most of the studies are in males (Pan et al., 2009). In the past decade, there have been several research findings adopting animal and human trials in treating PCOS, which is also an androgen-related disorder. It has been established that milled flaxseed consumption brings about a significant improvement in the regularity of the menstrual cycle (Oner, 2013).

In this present preliminary data, it was shown that there was a significant increase in the SHBG in the group supplemented with flax crackers. It was also observed that there was a significant reduction in the serum-free testosterone level with flax crackers. Total testosterone and serum insulin showed a trend towards improvement though statistically not significant. Also, an improvement in menstrual cycle regularity in the group supplemented with flax crackers was seen. The current study observed that the participants on flaxseed crackers showed a significant reduction in the FG score at the end of the study period, thus providing an ideal alternative to the drugs administered for the same.

The Total testosterone, Free testosterone, and Sex HormoneBinding Globulin (SHBG) levels were included as part of the clinical assessment of the female androgen levels in PCOS (Haidari et al., 2019).

Excessive androgen secretion in PCOS conditions causes an increase in the number of immature follicles and it is found that flaxseed significantly reduces serum androgen levels. It has been reported in previous research findings by Sturgeon SR et al. and Debra Novak et al. that a decline in testosterone, oestradiol, and estrone levels was observed following dietary flaxseed supplementation, especially in overweight/obese women (Debra et al., 2007, Sturgeon et al., 2008).

SHBG is a significant protein found to bind to some of the total testosterone, thereby reducing the free testosterone availability in blood circulation (Farzana et al., 2015) thus being an effective indicator for the determination of androgen levels. Lowered levels of SHBG indicate elevated active testosterone levels in the blood predisposing women to greater intensity of manifestation of clinical symptoms of acne, hirsutism, and male pattern alopecia. PCOS patients are therefore advised to take a diet that is rich in lignans and fiber to maintain optimum levels of SHBG in the blood and flaxseeds are a very good source of fiber and lignans. These seeds also contribute to high amounts of omega- 3 and omega- 6 
fatty acids proven to have very high anti-inflammatory properties, which regulate glucose metabolism and increased insulinsensitivity.

Previous studies have reported that there is an inverse relationship between SHBG and insulin levels, wherein insulin acts indirectly by decreasing the hepatic biosynthesis of SHBG (Khani et al. 2017). Furthermore, research findings have reported flaxseed or its isolated lignan (SDG) counterpart to reduce the insulin-like growth factor I (IGF-I) levels in the blood. Furthermore, it is reported by Sharon E et al. that consumption of flaxseed or its isolated lignan (SDG) reduces the insulin-like growth factor I (IGF-1) levels in the plasma found in rats with breast cancer (Rickarda et al., 2000). It has been found that IGF-1 increases LH secretion, which leads to hyperinsulinemia and lowers SHBG levels (Hara et al., 2012). Thus, a reduction in plasma IGF1 and subsequently insulin levels can lead to a decrease in LH secretion, causing an elevation in SHBG and lowering of testosterone. It has been established that the pharmaceutical drug metformin functions in lowering insulin by its impact on IGF-1 levels. So, there could be a possibility that flaxseed could bring about a similar change in the insulin levels in PCOS (Evanthia \& Farid, 2009). It has also been reported that metformin therapy increases circulating IGFBP-1 levels and thereby lowers the free-bioactive IGF-I in the blood circulation (Kolodziejczyk et al., 2000).

Elevation of testosterone leads to a male-pattern terminal hair growth pattern in females termed as hirsutism and is generally assessed using the $\mathrm{mFG}$ score, the most common diagnostic criterion of hyperandrogenism in clinical practice (Aswini \& Jayapalan, 2017). Anti-androgenic medications like flutamide blocks androgen receptors and finasteride prevent the conversion of testosterone to more active androgens, thereby facilitating a reduction in excess hair growth by lowering circulating androgens, however, though side effects are rare they have harmful effects on the liver (A Guide for Patients Revised American Society for reproductive medicine Hirsutism and Polycystic Ovary Syndrome (PCOS), 2016). On the contrary, flaxseeds have been proven; however, to reduce liver enzymes and improve the status of nonalcoholic liver disease, thus creating a protective role in maintaining the health of the liver (Yari et al., 2016).

\section{Conclusion}

Increased globalization has made women play a pivotal role in society. Their health status determines their productivity at home and work and the nation at large. With the increased economic independence of women, especially in the urban sector owing to their sedentary lifestyle coupled with affordable calorie-dense food, research in different cultural contexts for enhancing the productivity of women by improving their physical, emotional, and social well-being is imperative. It has also been proven that women belonging to the reproductive age are more susceptible to hormonal /endocrine imbalances and the most common being PCOS. In this randomized controlled clinical trial among young adult women, it has been found that milled flaxseeds baked as crackers for convenience of consumption and palatability have significant androgen and metabolic lowering potential ameliorating the clinical and biochemical symptoms in PCOS. Thus, in this present study, flaxseed crackers containing $30 \mathrm{~g}$ of milled flax powder taken for 5 months had beneficial effects on lowering the insulin levels, androgen, and sex hormone transporters, regularising menstrual cycle, and reducing $\mathrm{mFG}$ score, thereby decreasing hirsutism among young women. Considering this improvement in the metabolic and hormonal levels in PCOS diagnosed women and also lowering the clinical manifestation of hirsutism, flaxseeds can be incorporated for developing functional food traditional Indian products and can serve as an ideal alternative source of plant origin as a substitute for pharmaceutical formulations for the treatment of PCOS. The limitation of the present study was the small sample size; while the results were promising, the study necessitates validation with larger sample size

\section{Acknowledgments}

We acknowledge the contribution of biostatistician Dr. S. Xavier, Assistant Professor, Department of Statistics, Loyola College, Chennai, for the statistical analysis of the data in this study.

\section{Conflict of interest}

The authors declare that they have no conflict of interest.

\section{References}

A Guide for Patients Revised American Society for reproductive medicine Hirsutism and Polycystic Ovary Syndrome (PCOS). (2016): 9

Adlercreutz H, Höckerstedt K, Bannwart C, Bloigu S, Hämäläinen E, Fotsis T, Ollus A (1987) Effect of dietary components, including lignans and phytoestrogens, on enterohepatic circulation and liver metabolism of estrogens and on sex hormone binding globulin (SHBG). Journal of Steroid Biochemistry 27:1135-44.

Aswini R, Jayapalan S (2017) Modified Ferriman-Gallwey score in hirsutism and its association with metabolic syndrome. International Journal of Trichology 9:7-13.

Azziz R, Carmina E, Dewailly D, Diamanti-Kandarakis E, Escobar-Morreale HF, Futterweit W, Janssen OE, Legro RS, 
Norman RJ, Taylor AE, Witchel SF (2006) Position statement: Criteria for defining polycystic ovary syndrome as a predominantly hyperandrogenic syndrome: An androgen excess society guideline. Journal of Clinical Endocrinology 91:4237-4245.

Balaji S, Amadi C, Prasad S, Kasav JB, Upadhyay V, Singh AK, Surapaneni KM, Joshi A (2015) Urban rural comparisons of polycystic ovary syndrome burden among adolescent girls in a hospital setting in India. Biomedical Research International 158951:1-10.

Bloedon LT, Szapary PO (2004) Flaxseed and cardiovascular risk. Nutrition Review 62 (1):18-27.

Carraro JCC, Dantas MIS, Espeschit ACR, Martino HSD, Ribeiro SMR (2012) Flaxseed and human health: Reviewing benefits and adverse effects, Food Review International 28:03-230.

Chang AY, Oshiro J, Ayers C, Auchus RJ (2016) Influence of race/ethnicity on cardiovascular risk factors in polycystic ovary syndrome, the Dallas heart study. Clinical Endocrinology 85(1):92-99.

Costello M, Shrestha BB, Eden J, Sjoblom P, Johnson NN (2007) Insulin sensitizing drugs versus the combined oral contraceptive pill for hirsutism, acne and risk of diabetes, cardiovascular disease, and endometrial cancer in polycystic ovary syndrome. Cochrane Database System Review 24(1):1200-1209

Debra AN, Denise C, Snyder, Brown AJ, Wendy Wahnefried WD (2007) The effect of flaxseed supplementation on hormonal levels associated with polycystic ovarian syndrome: A case study. Current Top Nutraceutical Research 5(4):177-181.

Ebrahimi FA, Samimi M, Foroozanfard F, Jamilian M, Akbari H, Rahmani E, Ahmadi S, Taghizadeh M, Memarzadeh MR, Asemi Z (2017) The effects of Omega3 fatty acids and vitamin E cosupplementation on indices of insulin resistance and hormonal parameters in patients with polycystic ovary syndrome: A randomized, double-blind, placebo-controlled trial. Experimental and Clinical Endocrinology and Diabetes 125:353-359.

Escobar-Morreale HF, Luque-Ramırez M, San Millan JL (2005) The Molecular-genetic basis of functional hyperandrogenism and the polycystic ovary syndrome. Endocrine Reviews 26(2): 251282.

Evanthia DK, Farid NR (2009) Diagnosis and Management of Polycystic Ovarian Syndrome, Chapter 18, Springer US:209-232.

Farzana K, Sulaiman AF, Ruckmani A, Vijayalakshmi K, Lakshmi KG, Ranjini S, Duraivel M (2015) Effects of flax seeds supplementation in polycystic ovarian syndrome. International
Journal of Pharmaceutical Sciences Review and Research 31(1):113-119

Gambineri A, Pelusi C, Vicennati V, Pagotto U, Pasquali R (2002) Obesity and the polycystic ovary syndrome. International Journal of Obesity and Related Metabolic Disorders 26:883-896.

Ganie MA, Vasudevan V, Wani IA, Baba MS, Arif T, Rashid A (2019) Epidemiology, pathogenesis, genetics \& management of polycystic ovary syndrome in India. Indian Journal of Medical Research 150:333-344.

Goyal A, Sharma V, Upadhyay N, Gill Sihag M (2014) Flax and flaxseed oil: an ancient medicine \& modern functional food. Journal of Food Science and Technology 51(9):1633-1653.

Haidari F, Banaei-Jahromi N, Zakerkish M, Ahmadi K (2019) The effects of flaxseed supplementation on metabolic status in women with polycystic ovary syndrome: A randomized open-labelled controlled clinical trial. Nutrition Journal 19(8):1-11

Hara N, Takizawa, Isahaya E, Nishiyama T, Hoshii T, Ishizaki F, Takahashi K (2012) Insulin-like growth factor-1 is associated with regulation of the luteinizing hormone production in men receiving androgen deprivation therapy with gonadotropin-releasing hormone analogues for localized prostate cancer. Urologic Oncology 30(5):596-601.

Huang Z, Yong EL (2016) "Ethnic Differences: Is there an Asian Phenotype for Polycystic Ovarian Syndrome?" Best practice \& research. Clinical Obstetrics \& Gynecology 37:1-28.

Johnson NP (2014) Metformin use in women with polycystic ovarian syndrome. Annals of Translation Medicine 2(6):1-7.

Khani B, Mardanian F, Fesharaki SJ (2017) Omega-3 supplementation effects on polycystic ovary syndrome symptoms and metabolic syndrome. Journal of Research in Medical Sciences 22:644.

Kim JJ, Choi YM (2019) Phenotype and genotype of polycystic ovary syndrome in Asia: Ethnic differences. Journal of Obstetrics and Gynecology 45:2330-2337.

Kolodziejczyk B, Duleba AJ, Spaczynski RZ, Pawelczyk L (2000) Metformin therapy decreases hyperandrogenism and hyperinsulinemia in women with polycystic ovary syndrome. Fertility and Sterility 73(6):1149-1154.

Legro RS, Albanian SA, Ehrmann DA, Hoeger KM, Murad MH, Pasquali R, Welt CK (2013) Diagnosis and treatment of polycystic ovary syndrome: an endocrine society clinical practice guideline. Journal of Clinical and Endocrinology Metabolism 98:4565-92. 
Li R, Zhang Q, Yang D, Li S, Lu, S, Wu, X, Wang X, Fu S, Lin J, Zhu Y, Jiang Y, Feng HL, Qiao J (2013) Prevalence of polycystic ovary syndrome in women in China: A large community-based study. Human Reproduction 28:2562-2569.

Lipilina E, Ganji V (2017) Incorporation of ground flaxseed into bakery products and its effect on sensory and nutritional characteristics - a pilot study. Journal of Foodservice 20: 52-5924.

Low YL, Taylor JI, Grace PB, Dowsett M, Folkerd E, Doody D, Dunning AM, Scollen S, Mulligan AA, Welch AA (2005) Polymorphisms in the CYP19 gene may affect the positive correlations between serum and urine phytoestrogen metabolites and plasma androgen concentrations in men. Journal of Nutrition (135): 2680-2686.

Oner G (2013) Efficacy of omega-3 in the treatment of polycystic ovary syndrome. Journal of Obstetrics and Gynecology 33:289299.

Pan A, Sun J, Chen J, Ye X, Li H, Yu Z, Wang Y, Gu W, Zhang, $X$, Chen, X Wahnefried WD (2009) Effects of a flaxseed-derived lignan supplement on C-reactive protein, IL-6 and retinol-binding protein 4 in type 2 diabetic patients. British Journal of Nutrition 101:1145-1149.

Rahimlou M, Jahromi NB, Hasanyani N, Ahmadi AR (2019) Effects of flaxseed interventions on circulating inflammatory biomarkers: A systematic review and meta-analysis of randomized controlled trials. Advance Nutrition 10(6):1108-1119.

Rickarda SE, Yvonne V, Yuana B, Thompson LU (2000) Plasma insulin-like growth factor I levels in rats are reduced by dietary supplementation of flaxseed or its lignan secoisolariciresinol diglycoside. Cancer Letters 161(1):47-55.

Sartang MM, Mazloom Z, Dehkordi HR, Boldaji RB, Bellissimo N, Zepetnek JD (2017) The effect of flaxseed supplementation on body weight and body composition: A systematic review and meta-analysis of 45 randomized placebo-controlled trials. Obstetrics Review 18:1096-1107.
Sheelaa WG, Radha R (2014) The prevalence and features of the polycystic ovary syndrome in young South Indian women from Pondicherry, International Journal of Reproduction, Contraception, Obstetrics and Gynecology 3(2): 344-346.

Slavin JL (2005) Dietary fiber and weight. Nutrition 21(3): 411418.

Stein IF, Leventhal ML (1935) Amenorrhea associated with bilateral polycystic ovaries. American Journal of Obstetrics and Gynecology 29: 181-191.

Sturgeon SR, Heersink JL, Volpe SL, Bertone-Johnson ER, Puleo E, Stanczyk FZ, Sabelawski S, Wahala K, Kurzer MS, Bigelow C (2008) Effect of dietary flaxseed on serum levels of estrogens and androgens in postmenopausal women. Nutrition Cancer 60(5):612618.

Vidya Bharathia R, Swethaa S, Neerajaaa J, Varsha Madhavicaa J, Moorthy Janania D, Rekhaa SN, Ramyab S, Ushaa B (2017) An epidemiological survey: Effect of predisposing factors for PCOS in Indian urban and rural population. Middle East Fertility Society Journal 22:313-316.

Vos T, Flaxman AD, Naghavi M, Lozano R, Michaud C (2012) Years lived with disability (YLDs) for 1160 sequelae of 289 diseases and injuries 1990-2010: A systematic analysis for the Global Burden of Disease Study 2010. Lancet 380: 2163-2196.

Wang R, Mol BWJ (2017), The Rotterdam criteria for polycystic ovary syndrome: Evidence-based criteria? Human Reproduction $32: 261-264$

Yari Z, Rahimlou M, Eslamparast T, Daryani NE, Poustchi H, Hekmatdoost, A (2016) Flaxseed supplementation in non-alcoholic fatty liver disease: A pilot randomized, open labeled, controlled study. International Journal of Food Science and Nutrition 67:461469 